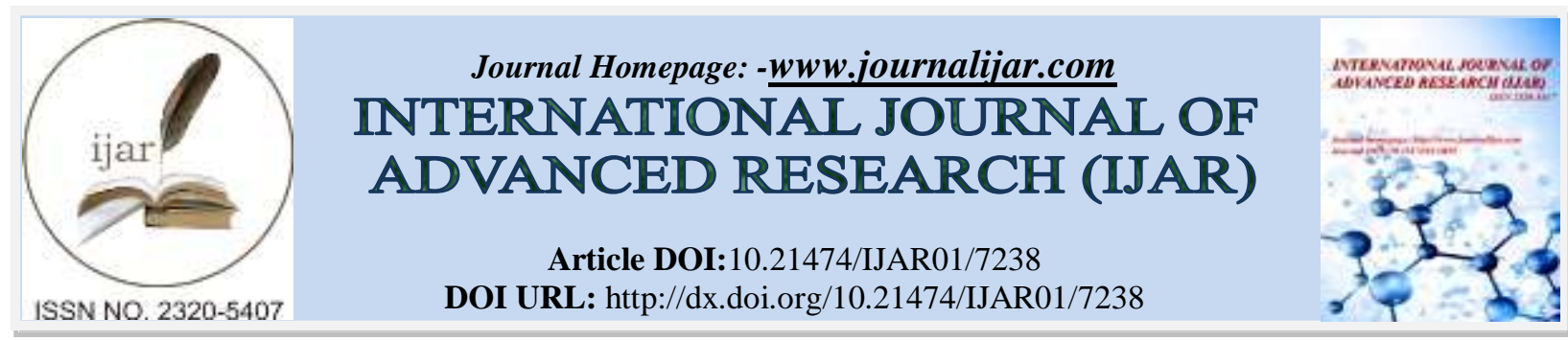

RESEARCH ARTICLE

\title{
SAFETY AND EFFICACY OF THALIDOMIDE IN PATIENTS WITH POEMS SYNDROME: A CASE REPORT.
}

Charifa Bassit, Jaouad Yousfi, Mouna Zahlane and Lamiaa Essaadouni.

Département of internal medicine, university hospitals Mohamed VI Marrakesh, Morrocco.

\section{Manuscript Info}

Manuscript History

Received: 09 April 2018

Final Accepted: 11 May 2018

Published: June 2018

Keywords:-

POEMS syndrome, thalidomide, efficacy.

\begin{abstract}
Polyneuropathy, Organomegaly, Endocrinopathy, M-protein, and Skin changes (POEMS) syndrome is a typical form of monoclonal gammapathy of clinical significance (MGCS). The acronym MGCS was coined by Jean-Paul Fermand and colleagues ${ }^{(1)}$ to generalise the notion of monoclonal gammapathy of renal significance, in which the toxicity of a monoclonal immunoglobulin produced by a B-cell clone is directed against various renal structures ${ }^{(2)}$. The first case of POEMS syndrome successfully treated with thalidomide was reported in $2004^{(3)}$. The authors then reported that thalidomide reduced serum VEGF concentrations and increased nerve conduction velocity in nine patients who were not suitable for autotransplantation because of advanced age or severe illness. We aimed to assess the efficacy and safety of thalidomide for the treatment of POEMS syndrome about one case.
\end{abstract}

Copy Right, IJAR, 2018,. All rights reserved.

\section{Case report:-}

Mrs. TK 62 years old, with no previous medical history except for a type 2 diabetes, addressed in our training for gait disorders .Neurological examination noted an erratic step of coordination with abolished osteotendinous reflexes to the two lower limbs with negative babinski sign. The abdominal examination showed a splenomegaly to two fingers through. The skin examination revealed an hyperpigmentation widespread.

The diagnosis of POEMS syndrome was based on the association of major and minor criteria:

\begin{tabular}{|c|c|}
\hline Major Criteria & Minor Criteria \\
\hline $\begin{array}{l}\text { - Severe demyelinating peripheral neurogenic } \\
\text { involvement }\end{array}$ & -Organomegaly: hepatosplenomegaly. \\
\hline $\begin{array}{l}\text { - Monoclonal gammopathy type IgG lambda at the } \\
\text { immunoelectrophoresis test. }\end{array}$ & $\begin{array}{l}\text { - Ascitic edematous syndrome: edema of the lower } \\
\text { limbs, pleural effusion, ascites. } \\
\text { - Endocrinopathy: diabetes, nodular goiter Tirads } 3 \text {. } \\
\text {-Cutaneous signs: hyperpigmentation. } \\
\text { - Lytic lesions in the pelvis, cranium and femur (spinal } \\
\text { exploration was without abnormalities) }\end{array}$ \\
\hline
\end{tabular}

The diagnosis was conforting with:

-Pulmonary arterial hypertension moderate to $47 \mathrm{mmhg}$.

-Renal insufficiency with GFR (glomerular filtration rate) at admission to 26.11.

- Osteomedullary biopsy was normal.

Address:-Département of internal medicine, university hospitals Mohamed VI Marrakesh, Morrocco. 
The treatment consisted of a corticosteroid dose of $1 \mathrm{mg} / \mathrm{kg} /$ day with a thalidomide $100 \mathrm{mg}$ oraly daily treatment. The evolution was marked by a good clinical improvement (regression of edema) and biological (increase of Renal function), with a follow-up of 18 months.

\section{Discussion:-}

Polyneuropathy, organomegaly, endocrinopathy, monoclonal gammopathy, and skin changes (POEMS) syndrome is a rare multisystemic disease that occurs in the setting of a plasma cell dyscrasia. The pathophysiologic link between the constellation of symptoms and the underlying disease is not well understood, but the link may be related to changes in the levels of a cytokine or a growth factor. POEMS syndrome was first described by Crow in 1956 and then by Fukase in 1968. The syndrome was termed Crow-Fukase syndrome (by which it is known in Japan) by Nakanishi in a study of 102 cases in Japan.

In 1980, the acronym POEMS was coined by Bardwick et al based on the 5 main features of the disease, namely, polyneuropathy, organomegaly, endocrinopathy, monoclonal gammopathy, and skin changes.

No specific case definition exists for POEMS syndrome; however, most authors agree that patients with POEMS syndrome should have 3 or more of the 5 features. Some authors have proposed that the presence of 2 major criteria, including a monoclonal plasma-proliferative disorder and polyneuropathy, in addition to the existence of 1 minor criteria, is sufficient for diagnosis. The suggested minor criteria include sclerotic bone lesions, organomegaly, edema, endocrinopathy, papilledema, and skin changes. However, the findings of a retrospective analysis of 629 patients using these criteria suggest that this approach may be inadequate for excluding other disease processes that may account for symptoms and that atypical presentations of POEMS may be misdiagnosed ${ }^{(4,5)}$

The treatment of POEMS syndrome depends on the treatment of the underlying plasma cell disorder. Most patients are treated with a combination of medical, surgical, and adjuvant therapies.

The current mainstays of treatment for patients with diffuse disease include combinations of corticosteroids, lowdose alkylators, and peripheral blood stem cell transplantation following high-dose chemotherapy. Some caution should be used in selecting the chemotherapeutic regimen to avoid worsening of the polyneuropathy.

Widespread osteosclerotic lesions may benefit from systemic therapy, with approximately half the patients benefiting from melphalan and prednisone. Although about a quarter of patients respond to corticosteroids, relapses are common without additional treatment of the underlying plasma cell disorder. Intravenous immunoglobulin and plasmapheresis have not shown therapeutic benefit.

In a study from China, 31 patients received 12 cycles of oral melphalan $\left(10 \mathrm{mg} / \mathrm{m}^{2}\right.$ body surface area [BSA] $)$ plus oral dexamethasone (40 mg/d) on days 1-4 of every 28 days. ${ }^{(6)}$ Prophylaxis with a proton-pump inhibitor was added days -1 to 5 . Doses were adjusted according to side effects and renal function. Good hematologic and neurologic responses were reported.

In a series of 30 POEMS syndrome patients treated at the Mayo Clinic in Rochester, Minn, engraftment syndrome was reported in approximately half the patients, with symptoms including fever (93\%), diarrhea (77\%), weight gain $(53 \%)$, and rash $(43 \%)$, in addition to a $3 \%$ treatment-related mortality rate. Baseline splenomegaly was predictive of a complicated peritransplantation course. ${ }^{(7)}$

Misawa and colleagues ${ }^{(8)}$ succeeded in showing, in the 13 patients who received dexamethasone and thalidomide, a greater reduction in VEGF concentration than in the 11 patients treated with dexamethasone and placebo (adjusted mean difference in VEGF concentration reduction rate $0 \cdot 41,95 \%$ CI $0 \cdot 02-0 \cdot 80 ; p=0 \cdot 04)$. However, because of the small number of patients, many of the comparisons were done on a very small population; for example, only four of eight patients in the thalidomide group and one of five in the placebo group achieved a normal serum VEGF concentration at 24 weeks. For neurological clinical and laboratory endpoints, the effect of dexamethasone and thalidomide was far from impressive compared with dexamethasone and placebo. For example, the difference in motor sum score was statistically significant $(\mathrm{p}=0.048)$, but the difference of 3.01 points (95\% CI $0.03-5 \cdot 99)$ clearly represents a marginal improvement and unfortunately did not translate into functional improvement (as measured by the overall neuropathy limitation scale), which would be a better marker of treatment efficacy. 
The study by Misawa and colleagues ${ }^{(8)}$ does not define a new standard of care in POEMS syndrome, but confirms the interest of using immunomodulatory drugs for POEMS syndrome and clearly shows that prospective and even randomised trials are feasible in this rare disease.

Thalidomide represents a new treatment option for patients with POEMS syndrome who are not eligible for transplantation, findings from many observational studies have shown positive effects of treatment with thalidomide ${ }^{(9,10)}$ lenalidomide ${ }^{(11)}$ low-dose of alkylators ${ }^{(12,13)}$ and bortezomib ${ }^{(14)}$ However, there is no established treatment for POEMS syndrome.

Thalidomide has many actions potentially useful for the treatment of POEMS syndrome, including suppression of monoclonal plasma cell proliferation and modulation of upregulated cytokines ${ }^{(15)}$. Although increased serum VEGF concentrations presumably play a major part in the pathogenesis of POEMS syndrome, other pro-inflammatory cytokines, such as tumour necrosis factor- $\alpha$ (TNF- $\alpha$ ) and interleukins 6 and 12, are also markedly upregulated and seem to be involved. These inflammatory cytokines decrease after transplantation or thalidomide treatment. We speculate that several mechanisms of thalidomide action contribute to the positive effects on POEMS syndrome. In our patient the improvement was spectacular evolution with a low dose of thalidomide.

\section{Conclusion:-}

POEMS syndrome is a potentially fatal disease with severe polyneuropathy and multi-organ involvement. The worldwide occurrence of this disease has become apparent over the past two decades. Thalidomide reduces serum VEGF concentrations and represents a new treatment for patients with POEMS syndrome who are not eligible for stem-cell transplantation.

\section{Bibliography:-}

1. Nelson Leung,1,2 Frank Bridoux, on behalf of the International Kidney and Monoclonal Gammopathy Research Group online October 9, 2012 doi:10.1182/blood-2012-07-445304 originally published .

2. Merlini G, Stone MJ. Dangerous small B-cell clones. Blood 2006; 108: 2520-30.

3. Sinisalo M, Hietaharju A, Sauranen J, Wirta O. Thalidomide in POEMS syndrome: case report. Am J Hematol 2004; 76: 66-68.

4. Ofran Y, Yishay O, Elinav E, Eran E. POEMS syndrome: failure of newly suggested diagnostic criteria to anticipate the development of the syndrome. Am J Hematol. 2005 Aug. 79(4):316-8.

5. Morizane R, Sasamura H, Minakuchi H, Takae Y, Kikuchi H, Yoshiya N, et al. A case of atypical POEMS syndrome without polyneuropathy. Eur J Haematol. 2008 May. 80(5):452-5.

6. Li J, Zhang W, Jiao L, Duan MH, Guan HZ, Zhu WG, et al. Combination of melphalan and dexamethasone for patients with newly diagnosed POEMS syndrome. Blood. 2011 Mar 10.

7. Dispenzieri A, Lacy MQ, Hayman SR, Kumar SK, Buadi F, Dingli D, et al. Peripheral blood stem cell transplant for POEMS syndrome is associated with high rates of engraftment syndrome. Eur J Haematol. 2008 May. 80(5):397406.

8. Misawa S, Sato Y, Katayama K, For the Japanese POEMS Syndrome for Thalidomide (J-POST) Trial Study Group. Safety and efficacy of thalidomide in patients with POEMS syndrome: a multicentre, randomised, double-blind, placebo-controlled trial. Lancet Neurol 2016; published online Aug 2. S1474-4422(16).

9. Kuwabara S, Misawa S, Kanai K, et al. Thalidomide reduces serum VEGF levels and improves peripheral neuropathy in POEMS syndrome. J NeurolNeurosurg Psychiatry 2008; 79: 1255-57.

10. Inoue D, Kato A, Tabata S, Successful treatment of POEMS syndrome complicated by severe congestive heart failure with thalidomide. Intern Med 2010; 49: 461-66.

11. Royer B, Merlusca L, Abraham J, Efficacy of lenalidomide in POEMS syndrome: a retrospective study of 20 patients. Am J Hematol 2013; 88: 207-12.

12. Kuwabara S, Hattori T, Shimoe Y, Kamitsukasa I. Long term melphalan-prednisolone chemotherapy for POEMS syndrome. J NeurolNeurosurg Psychiatry 1997; 63: 385-87.

13. Li J, Zhang W, Jiao L, Combination of melphalan and dexamethasone for patients with newly diagnosed POEMS syndrome. Blood 2011; 117: 6445-49.

14. Tang X, Shi X, Sun A, Successful bortezomib-based treatment in POEMS syndrome. Eur J Haematol 2009; 83: 60910 .

15. Gordon JN, Goggin PM. Thalidomide and its derivatives: emerging from the wilderness. Postgrad Med J 2003; 79: $127-32$. 